\title{
Neutron Cross Section Measurements at the Spallation Neutron Source
}

\author{
Klaus H. GUBER*, Paul E. KOEHLER, Timothy E. VALENTINE, and Luiz C. LEAL
}

Oak Ridge National Laboratory, P.O. Box 2008, Oak Ridge, TN 37831-6354, USA

\begin{abstract}
With the prospect of construction of the Spallation Neutron Source (SNS) at ORNL, and the fantastic high neutron flux, new, up to now impossible, experiments seem to be feasible in the fields of applied nuclear physics and astrophysics. These experiments will supply crucial neutron-induced cross section data for radionuclides, which are badly needed by many applied physics programs. The SNS will be uniquely suited for measuring the cross sections of interest to nuclear criticality safety, accelerator transmutation of nuclear waste (ATW), and heavy element nucleosynthesis for astrophysics. Because the sample sizes required at current facilities are usually too large for practical measurements, scarce information of these cross sections is available. Using the high neutron flux at the SNS will allow these measurements to be made with samples about 40 times smaller than at the next best facility. The large reduction in sample size at the SNS will result in orders of magnitude reduction in background from the radioactive samples and make them much easier to produce; hence, a much wider range of samples will be accessible for measurement at the SNS than at any other facility.
\end{abstract}

Keywords: neutron cross section, SNS, small samples, radioactive samples

\section{Introduction}

Using the extremely high neutron flux at the SNS will enable new, up to now impossible, experiments in the field of neutron physics by allowing neutron induced cross section measurements from thermal neutron energies up to several $\mathrm{keV}$ on very small samples. There are many cross section measurements of importance to nuclear and applied physics programs in the areas of nuclear astrophysics, nuclear data, and criticality safety requiring the use of radioactive samples. More detailed studies revealed a significant lack of nuclear data. ${ }^{1}$ In nuclear astrophysics, these new data will lead to a better understanding of the origin of the nuclides heavier than iron, help to explain the mysteries of the origin of isotopic anomalies in meteorites, and provide "benchmarks" to understand the inner workings of stars and supernovae. An established nuclear data program would advance the basic research and instrument science, which are the foundations for many DOE's applied physics programs. For example modern reactor design requires new and more accurate nuclear data not only for uranium and transuranium isotopes but also for long lived fission products (neutron poisons). In addition, these nuclear data will be useful to provide fundamental information to address the possibility of waste transmutation. This will directly address the needs of the Basic Energy Research in a safe energy generation, conversion, transmission, and use.

Because the sample sizes required at current facilities are usually too large for practical measurements, scarce information about these cross sections is available. The high neutron flux at the SNS should allow these measurements to be made with samples about 40 times smaller than at the next best facility. ${ }^{2)}$ This large reduction in sample size will result in orders of magnitude reduction in background from the radioactive samples and make them much easier to produce. Hence, measurements can be performed on a much wider range of samples at the SNS than at any other facility.

\section{Experimental Approach}

The SNS (Fig. 1) basically consists of three parts. First, a high-energy and -powered proton accelerator $(\sim 1 \mathrm{GeV}$ and 2 MW) with $60 \mathrm{~Hz}$ repetition rate and less than $1 \mu$ s pulse width. Second, a target system including the Hg-target, moderator, reflector, shielding and shutter. The third part consists of the experimental stations and beam lines. At present there will be 18 beam line and shutters, which view the four different moderators.

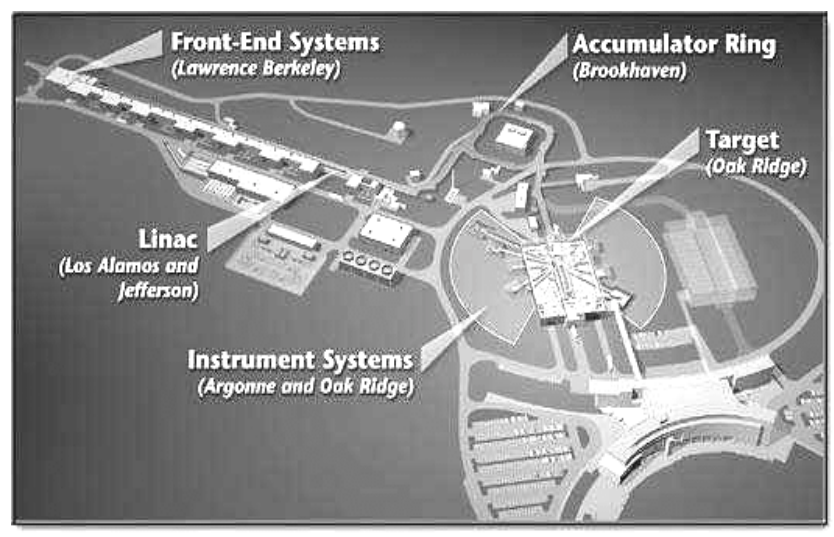

Fig. 1 Schematic view of the Spallation Neutron Source at ORNL.

\footnotetext{
${ }^{*}$ Corresponding author, Tel. +865-574-6117, Fax. +865-576-8746

E-mail: guberkh@ornl.gov
} 
Advanced detection systems will be required for the measurement of a variety of neutron-induced cross sections such as total, inelastic scattering, $(n, \gamma),(n, p),(n, \alpha)$, and fission. For example, new designs for ionization chambers or particle detectors are required in order to use very small samples in a high background environment. In addition, careful studies and simulations are necessary to ascertain how to overcome the conflicting requirements for $(n, \gamma)$ measurements, such as high efficiency for $\gamma$ rays, low sensitivity to the backgrounds from the decay of the radioactive sample, as well as from sample scattered neutrons. Previous measurements with Barium fluoride, ${ }^{3)}$ and deuterated benzene ${ }^{4)}$ show that these materials seem to be feasible as detector materials. Recent experiments ${ }^{5,6)}$ with a much weaker neutron flux demonstrated impressively that $(\mathrm{n}, \gamma)$ measurements on radioactive and very small samples $(8$ $\mathrm{mg}$ ) are possible by using a $4 \pi-\mathrm{BaF}_{2}$ detector (Fig. 2). ${ }^{7)}$ Since these detectors also have drawbacks, further Monte Carlo simulations as well as actual performance tests of prototype detectors will be needed before the final detector can be designed with confidence. We plan to carry out these tests using the pulsed-white-neutron-source facility Oak Ridge Electron Linear Accelerator (ORELA). The excellent time-of-flight resolution available at ORELA will greatly facilitate these tests. Another experimental challenge is related to the multi-user character of most spallation facilities. Most of the instruments at these facilities are designed for neutrons near thermal energy; hence, the beam lines and instruments are not particularly well shielded for high-energy neutrons. In addition, beam choppers scatter a significant portion of the beam, and iron is a commonly employed shielding material. As a result, other beam lines constitute a significant source of background of relatively high-energy $\gamma$ rays.

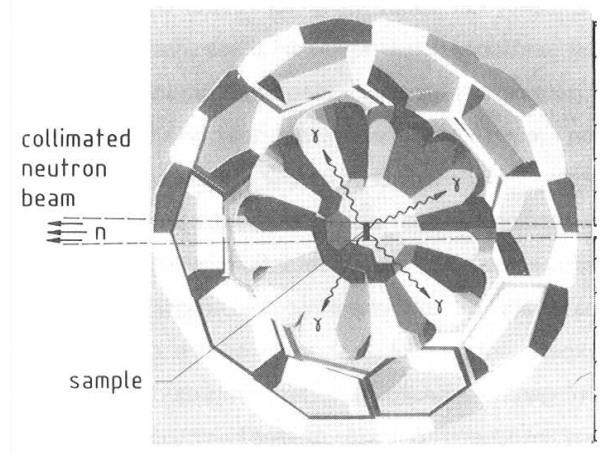

Fig. 2 Schematic view of the experimental set up used in Ref. 5 and 6 .

\section{Comparison with other Neutron Facilities}

In a recent publication, a comparison ${ }^{2)}$ was made of various white neutron sources for measurements using radioactive samples. These results are compiled in Table 1, for the Los Alamos Neutron Science Center (LANSCE), the CERN n_TOF, ${ }^{1,9)}$ and the Oak Ridge Spallation Neutron Source $(\mathrm{SNS})^{10)}$ which are compared to the ORELA facility. ${ }^{11)}$ Over the past three decades most previous $(n, \gamma)$ measurements have been made at ORELA. Typically, ORELA operating conditions for $(n, \gamma)$ measurements are 8 $\mathrm{kW}$ power, $8 \mathrm{~ns}$ electron pulse width and $40 \mathrm{~m}$ flight path length. However, neutron capture measurements at ORELA have been made at flight paths as short as $10 \mathrm{~m}$ and the facility has run for extended periods at powers as high as 50 $\mathrm{kW}$; hence, these conditions are also included in Table 1. At the LANSCE facility a flight path length of $20 \mathrm{~m}$ has been chosen $^{12)}$ for the new $(n, \gamma)$ instrument. In the past this facility has reliably operated at a power of $64 \mathrm{~kW}$. The CERN n_TOF facility was originally designed with detector stations at 80 and $230 \mathrm{~m}$, although currently a flight path length of $180 \mathrm{~m}$ is used. Under the most optimistic conditions, the power of this facility is $45 \mathrm{~kW}$. For a comparison with the SNS, a flight path length of $20 \mathrm{~m}$ was chosen because this would yield a time-of-flight resolution equivalent to previous nuclear astrophysics measurements at LANSCE.

Table 1 Ratio to the benchmark facility (ORELA at $8 \mathrm{~kW}, 8 \mathrm{~ns}$ pulse width, and $40 \mathrm{~m}$ flight path length) and estimated sample sizes.

\begin{tabular}{ccccccc} 
Parameter & \multicolumn{5}{c}{ Ratio to ORELA Benchmark } \\
\cline { 2 - 7 } & ORELA & LANSCE & \multicolumn{2}{c}{ CERN n_TOF } & SNS \\
\hline $\begin{array}{c}\text { Fight Path } \\
\text { Length }^{\mathrm{a}}(\mathrm{m})\end{array}$ & 10 & 40 & 20 & 230 & 80 & 20 \\
\hline Power $(\mathrm{kW})^{2}$ & 6.2 & 1 & 8 & 5.6 & 5.6 & 250 \\
\hline $\begin{array}{c}\text { Flux at } \\
30 \mathrm{keV}\end{array}$ & 100 & 1 & 280 & 10 & 85 & 12000 \\
\hline $\begin{array}{c}\text { Integral Flux } \\
1-300 \mathrm{keV}\end{array}$ & 100 & 1 & 230 & 9.5 & 75 & 10500 \\
\hline $\begin{array}{c}\text { Peak Flux } \\
1-300 \mathrm{keV}\end{array}$ & 210 & 1 & 12000 & 520 & 12000 & 180000 \\
\hline $1 /$ Puse width & 0.083 & 1 & 0.032 & 6.7 & 2.3 & 0.011 \\
\hline $\begin{array}{c}\text { Sample size } \\
\text { (mg) }\end{array}$ & 0.25 & 25 & 0.10 & 2.5 & 0.31 & 0.0022 \\
\hline
\end{tabular}

${ }^{a}$ The flight path length is given to define the facility. All other parameters except the sample size are ratio to the benchmark facility.

${ }^{\mathrm{b}}$ The sample size is scaled to cross section of $1 \mathrm{~b}$ and an atomic number of 150 , and assumes a detector efficiency of $100 \%$.

For measurements using small samples, the neutron flux is the most important facility parameter because it determines the size of the sample needed. For experiments using radioactive samples, the peak flux could be important in overcoming the background from the decay of the sample. The neutron fluxes in Table 1 are expected to be higher than that of the ORELA benchmark by factors of 10 to 11000 . Furthermore, the peak fluxes should be larger by factors of 500 to 180000 . Using the $10 \mathrm{~m}$ flight path and $50 \mathrm{~kW}$ power at ORELA, the flux could be as much as 100 times larger and the peak flux as much as 200 times larger than the ORELA benchmark. At present, only the ORELA and LANSCE facilities exist and are under routine operation. The LANSCE beam line for $(n, \gamma)$ measurements currently is 
being rebuilt. The CERN n_TOF facility performed their first test measurements in May and June 2001. The first analysis of their results show serious background problems related to high energy particles from the spallation target. The SNS is under construction and is on schedule to be completed in 2006.

\section{Samples}

There are a large number of isotopes, which are of interest to the nuclear astrophysics community. These mainly involve the understanding of the nucleosynthesis of the heavy elements, which are formed by three different processes ( $s, r$ and $p$-process $)$ in various stellar environments.

The $s$-process is the best understood, because most isotopes involved are stable and the $(n, \gamma)$ reaction rates have been measured. However, there remain a number of important nuclides in the $s$-process path for which no measurements have been made or for which previous measurements are unsatisfactory. A large number of the needed reaction rates are for radioactive isotopes that are branching points in the $s$-process flow where the half-lives are long enough so that neutron capture can compete with $\beta$ decay. These branching points offer the potential for very direct diagnostics of both the average properties as well as the "dynamics" of the s-process environment. Branching points in the $s$-process path for which measurements are needed include ${ }^{85} \mathrm{Kr},{ }^{134,135} \mathrm{Cs},{ }^{147} \mathrm{Nd},{ }^{147,148} \mathrm{Pm},{ }^{151} \mathrm{Sm},{ }^{152} \mathrm{Eu}$, ${ }^{153} \mathrm{Gd},{ }^{163} \mathrm{Dy},{ }^{163,164} \mathrm{Ho},{ }^{169} \mathrm{Er},{ }^{170,171} \mathrm{Tm},{ }^{176} \mathrm{Lu},{ }^{185} \mathrm{~W}$, and ${ }^{186} \mathrm{Re}$.

In Table 2 we give estimates of the sample sizes needed for $(n, \gamma)$ measurements at the SNS. The sample sizes have been scaled using information from previous measurements at the benchmark facility. They would result in the same "sample in" counting rate as at the benchmark facility. This was done by assuming a $100 \%$ efficient detector. These estimates indicate that it should be possible to measure many of the $(n, \gamma)$ reaction rates for radioisotopes of interest to astrophysics at the SNS. Many of these elements are longlived fission products. Therefore they are also of interest to applied physics programs, since some of them are neutron poisons or otherwise are needed as input for criticality safety calculations.

The $r$-process most likely occurs during stellar explosions. The resulting high neutron density and temperature drive the nucleosythesis path far away from the valley of beta stability to the neutron rich side, where the process is in equilibrium. However, at the end of the $r$ process, as the temperature and neutron density decline, the reaction rates can become important. During this so-called freeze-out phase, reaction rate effects may help to smooth out pronounced odd-even effects predicted for the $r$-process yields. Although the freeze-out isotopes cannot be measured directly, the statistical model calculation could be improved by better input parameters. For this reason, reaction rate measurements on the following nuclides are needed: ${ }^{90} \mathrm{Sr}$, ${ }^{123} \mathrm{Sn},{ }^{126} \mathrm{Sn},{ }^{127 \mathrm{~m}} \mathrm{Te},{ }^{182} \mathrm{Hf},{ }^{210} \mathrm{~Pb},{ }^{226} \mathrm{Ra}$, and ${ }^{227} \mathrm{Ac}$.

Table 2 Possible candidates for $(\mathrm{n}, \gamma)$ cross section measurements in the neutron energy range from $100 \mathrm{eV}$ to $300 \mathrm{keV}$. These radionuclide are involved in $s$-process branchings.

\begin{tabular}{cccc}
\hline Isotope & $\begin{array}{c}\langle\sigma\rangle_{30}{ }^{\mathrm{a}} \\
(\mathrm{mb})\end{array}$ & $\mathrm{t}_{1 / 2}$ & $\begin{array}{c}\text { Atoms needed } \\
\text { at the SNS }\end{array}$ \\
\hline${ }^{79} \mathrm{Se}$ & 220 & $<6.510^{4} \mathrm{yr}$ & $4.510^{16}$ \\
${ }^{85} \mathrm{Kr}$ & 70 & $10.7 \mathrm{yr}$ & $1.510^{17}$ \\
${ }^{94} \mathrm{Nb}$ & 500 & $210^{4} \mathrm{y}$ & $2.010^{16}$ \\
${ }^{106} \mathrm{Ru}$ & 140 & $367 \mathrm{~d}$ & $7.110^{16}$ \\
${ }^{135} \mathrm{Cs}$ & 200 & $210^{6} \mathrm{y}$ & $5.010^{16}$ \\
${ }^{147} \mathrm{Pm}$ & 1200 & $2.62 \mathrm{yr}$ & $8.610^{15}$ \\
${ }^{155} \mathrm{Eu}$ & 1900 & $4.9 \mathrm{yr}$ & $5.310^{15}$ \\
${ }^{153} \mathrm{Gd}$ & 2800 & $241.6 \mathrm{~d}$ & $3.610^{15}$ \\
${ }^{163} \mathrm{Ho}$ & 2600 & $4570 \mathrm{yr}$ & $3.810^{15}$ \\
${ }^{169} \mathrm{Er}$ & 610 & $9.40 \mathrm{~d}$ & $1.610^{16}$ \\
${ }^{170} \mathrm{Tm}$ & 2300 & $128.6 \mathrm{~d}$ & $4.410^{15}$ \\
${ }^{171} \mathrm{Tm}$ & 990 & $1.92 \mathrm{yr}$ & $1.010^{16}$ \\
${ }^{179} \mathrm{Ta}$ & 1200 & $1.7 \mathrm{yr}$ & $8.310^{15}$ \\
${ }^{182} \mathrm{Hf}$ & 240 & $910^{6} \mathrm{y}$ & $4.210^{16}$ \\
${ }^{185} \mathrm{Hf}$ & 620 & $75.1 \mathrm{~d}$ & $1.610^{16}$ \\
${ }^{193} \mathrm{Pt}$ & 1500 & $50 \mathrm{yr}$ & $6.710^{15}$ \\
${ }^{204} \mathrm{Tl}$ & 130 & $3.77 \mathrm{yr}$ & $7.710^{16}$ \\
\hline
\end{tabular}

${ }^{\mathrm{a}}$ The $30 \mathrm{keV}$ reaction rates are based on calculations.

There are about 30 proton-rich isotopes of the intermediate to heavy mass elements that cannot be reached via neutron-capture nucleosynthesis. These are the so-called $p$ isotopes, and they are believed to be produced by photodisintegration starting from the $s$ process seed material. Calculations indicate that the high temperature of explosive burning in the $\mathrm{Ne} / \mathrm{O}$ shells of massive stars result in a series of $(\gamma, n),(\gamma, \alpha)$, and $(\gamma, p)$ reactions that convert the $s$-process material to the $p$ isotopes. In addition, during the freeze-out phase near the end of the $p$ process, $(n, \gamma)$ reactions have a strong impact on the final abundance distribution. Furthermore, $(n, \gamma)$ cross section measurements on proton rich nuclides could be used to calculate the needed $(\gamma, n)$ rates using detailed balance and to improve the statistical model for the calculation of rates that cannot be measured. Radioactive nuclides of interest to the $p$ process include ${ }^{53} \mathrm{Mn},{ }^{55} \mathrm{Fe},{ }^{57} \mathrm{Co},{ }^{59} \mathrm{Ni},{ }^{91,92} \mathrm{Nb},{ }^{93} \mathrm{Mo},{ }^{97} \mathrm{Tc},{ }^{109} \mathrm{Cd},{ }^{137} \mathrm{La}$, ${ }^{139} \mathrm{Ce},{ }^{143,145} \mathrm{Pm},{ }^{145,146} \mathrm{Sm},{ }^{148,150} \mathrm{Gd},{ }^{154,159} \mathrm{Dy},{ }^{157} \mathrm{~Tb},{ }^{172} \mathrm{Hf}$, ${ }^{195} \mathrm{Au},{ }^{194} \mathrm{Hg}$, and ${ }^{202} \mathrm{~Pb}$. In addition, $(n, \gamma)$ measurements are needed on the $p$ isotopes themselves. The sample sizes are small enough that the necessary isotopically enriched samples should be affordable for many of the $p$ isotopes.

The largest nuclear physics uncertainties affecting $p$ process calculations are the rates for $(\gamma, \alpha)$ reactions. A series of $(n, \alpha)$ measurements for $\mathrm{E}_{\mathrm{n}} \approx 1 \mathrm{eV}$ to $1 \mathrm{MeV}$ appears to offer the best hope to obtain global improvements in these rates. The first $(n, \alpha)$ measurements across the range of energies needed for astrophysics applications have recently been reported. $^{13)}$ Significant differences between the measurements and the latest statistical models have been observed. More data of this type are needed to obtain the 
necessary global constraints. The measurements are difficult because the cross sections are small ( $20 \mu \mathrm{b}$ or less); hence, because of their large fluxes, spallation sources are potentially very useful for future measurements of this type.

\section{Conclusion}

Spallation neutron sources have much potential for future applied physics and nuclear astrophysics experiments. With experiments at these facilities, there are likely to be a number of challenges to overcome before they fulfill their promise. The most difficult problems to overcome are related to radioactive samples and high peak fluxes.

\section{Acknowledgements}

This research is sponsored by the Office of Environmental Management, U.S. Department of Energy, under contract No. DE-AC05-00OR22725 with UT-Battelle, LLC.

\section{References}

1. Rubbia, C. et al., Technical report, European Laboratory for Particle Physics Report CERN/LHC/90-02 (EET), 1998; CERN/SPSC 99-8 SPSC/P 310, 1999.

2. P.E. Koehler, Nucl Instr. Meth. A460 (2001) 352.

3. K.H. Guber, R.R. Spencer, P.E. Koehler and R.R. Winters, Nuclear Physics A261 (1997), p. 254c-257c.

4. P.E. Koehler, R.R. Spencer, R. R. Winters, K.H. Guber, , J.A. Harvey, N.W. Hill and M.S. Smith, Phys. Rev. C 54, 1463 (1996)
5. K. Wisshak, F. Voss and F. Käppeler, Nucl. Sci. Eng., 137, 1 (2001).

6. K. Wisshak, et al., Forschungszentrum Karlsruhe, Germany, FZKA 6362.

7. K. Wisshak, K. Guber, F. Käppeler, J. Krisch, H. Müller, G. Rupp and F. Voss, Nucl. Instr. Meth. A292, 595 (1990)

8. Lisowski, P. W., Bowman, C. D., Russell, G. J., and Wender, S. A., The Los Alamos National Laboratory spallation neutron sources, Nucl. Sci. Eng. 106 (1990) 208-218.

9. C. Rubbia et al., "High Resolution Spallation Driven Facility at the CERN-PS to Measure neutron Cross Sections in the Interval from $1 \mathrm{eV}$ to $250 \mathrm{MeV}$ : A relative Performance Assessment”, CERN/LHC/98-02 (EET)Add. 1.

10. Olsen, D. et al., Technical report, Oak Ridge National Laboratory Report SNS 100000000PL0001R00, 1999.

11. Peelle, R. W., Harvey, J. A., Maienschein, F. C., Weston, L. W., Olsen, D. K., Larson, D. C. and Macklin, R. L., Technical report, Oak Ridge National Laboratory No. ORNL/TM-8225.

12. Ullmann, J. L., Haight, R. C., Fowler, M. M., Miller, G. G., Rundberg, R. S. and Wilhelmy, J. B., Neutron capture measurements on unstable nuclei at LANSCE, in Proceedings of the Fifteenth International Conference on the Application of Accelerators in Research and Industry, edited by J. L. Guggan and I. L. Morgan (American Institute of Physics, New York, 1999) pp. 251-254.

13. Y.M. Gledenov, P.E. Koehler, J. Andrzejewski, K.H. Guber, and T. Rauscher, Phys. Rev. C 62, 042801(R) (2000). 